\title{
Standardization of Drip Irrigation Scheduling for Jasmine (Jasminum sambac Ait. cv. Ramanathapuram Gundumalli) using Theta Probe moisture meter
}

\author{
S. Kumaresan* and M. Jawaharlal \\ Department of Floriculture and Landscaping, Horticultural College and Research Institute, Tamil \\ Nadu Agricultural University, Coimbatore- 641 003, Tamil Nadu, India \\ *Corresponding author
}

\begin{tabular}{|c|c|}
\hline & A B S T R A C T \\
\hline & \multirow{5}{*}{$\begin{array}{l}\text { An experiment entitled, "Standardization of drip irrigation scheduling for Jasmine } \\
\text { (Jasminum sambac Ait. cv. Ramanathapuram Gundumalli) using Theta Probe moisture } \\
\text { meter" was carried out at Farmer's holding, Neithalur Colony in Tiruchirappalli District, } \\
\text { Tamil Nadu, India from November, } 2015 \text { to May, } 2016 \text { to standardize the irrigation } \\
\text { requirement and irrigation frequency for Jasmine. Factorial randomized block design was } \\
\text { employed with three factors each two levels and replicated thrice. The investigation } \\
\text { consisted of two levels each on soil depth, moisture depletion percentage and quantity of } \\
\text { irrigation water viz., soil depth @ } 15 \mathrm{~cm} \text { and } 30 \mathrm{~cm} \text {, moisture percentage } 40 \% \text { and } 60 \% \\
\text { and quantity of irrigation water } 151 \text { and } 201 \text { per plant and flood irrigation (control). The } \\
\text { jasmine plants receiving } 201 \text { of irrigation water per plant when moisture depletion } \\
\text { percentage reaches } 60 \% \text { at } 30 \mathrm{~cm} \text { soil depth were performing well in all the growth, yield } \\
\text { and agronomic attributes. Hence, it is recommended that, irrigation @ } 201 \text { per plant at } 4 \\
\text { day intervals can be adopted for high growth, physiological, yield and agronomic } \\
\text { parameters in Jasmine plants. }\end{array}$} \\
\hline Keywords & \\
\hline $\begin{array}{l}\text { moisture } \\
\text { ge, } \\
\text { frequency } \\
\text { er yield }\end{array}$ & \\
\hline Ar & \\
\hline $\begin{array}{l}A c \\
30 \\
A\end{array}$ & \\
\hline
\end{tabular}

\section{Introduction}

The genus Jasminum belongs to the family Oleaceae. Although more than 2,000 species are known, 40 species have been identified in India and 20 are cultivated in South India (Bhattacharjee, 1980) which comprises mainly shrubs and climbers. Jasmine is a fragrance and an important commercial flower crop in tropical and sub-tropical areas of South East Asia and other parts of world. It is also an important source of jasmine concrete and perfume extraction. Jasmine is the national flower of Phillipines, which was adopted in 1937 (Sushma et al., 2015). And, recently in 1990, Indonesian government also has adopted it as the national flower (Jayamma et al., 2014). Jasmine concrete is now being produced industrially in India and it is a highly profitable venture and the demand is increasing in the world market because of its unique fragrance which cannot be imitated by synthetic chemicals.

The standard soil moisture estimation procedures such as gravimetric and neutron probe methods used were not well suited for portable use in field data collection. Recently, 
an impedance probe (Theta Probe, Delta-T Devices, Cambridge UK) has received acceptance for surface soil water content measurements, especially by the remote sensing community (Jacobs et al., 2004). A reliable, rapid technique is needed, and recently an impedance soil moisture probe (Theta Probe) has been accepted by the scientific community. Judicial management of irrigation water can be achieved by using soil moisture sensors like neutron probe and Theta Probe.

Many of the research works has been carried out on responses of jasmine plants to macro and micronutrients, plant growth and irrigation intervals but irrigation based on moisture depletion percentage and evapotranspiration has not been studied. With this above background the present study has been taken to standardize the irrigation requirement and its frequency using Theta Probe moisture meter for jasmine (Jasminum sambac).

\section{Materials and Methods}

Moisture depletion percentage was estimated in the soil profile $0-15 \mathrm{~cm}, 15-30 \mathrm{~cm}$ and 30 $45 \mathrm{~cm}$. The soil moisture depletion was more pronounced in the layer of 15-30 and 30 -45 $\mathrm{cm}$. So it was correlated with the evaporation and moisture extraction capacity of the jasmine plants. It was indicated that active plant roots are actively extracting the moisture content present in the layer of 15-45 $\mathrm{cm}$ than the layer of $0-15 \mathrm{~cm}$. The moisture content in the top layer $0-15 \mathrm{~cm}$ can be easily reduced due to prevailing environment, it was not affected the growth and development of jasmine.

The experiment was conducted at farmer's field, Neithalur Colony, Tiruchirappalli District, Tamil Nadu during last week of November, 2015 to May, 2016. Initial soil- chemical, physical properties of selected field were: $\mathrm{pH} 7.8$, EC $0.12 \mathrm{~d} \mathrm{sm}^{-1}, \mathrm{~N} 220 \mathrm{~kg} \mathrm{ha}^{-1}$, $\mathrm{P} 20.1 \mathrm{~kg} \mathrm{ha}^{-1}$, $\mathrm{K}_{2} 29 \mathrm{~kg} \mathrm{ha}^{-1}$ and bulk density of $1.36 \mathrm{~kg} \mathrm{~m}^{-3}$.

\section{Experiment Details}

The trial was laid out in Factorial Randomized Block Design with 8 treatment combinations and replicated thrice. The three factors having 2 levels such as 8 treatment combinations viz., two levels of irrigation water given (15 1 and 201 per plant) when soil moisture depletion reaches $40 \%$ and $60 \%$ at the depth of $15 \mathrm{~cm}$ and $30 \mathrm{~cm}$ as per the treatment schedule and control (Flood irrigation once in a week). The jasmine bushes were uniformly pruned during last week of November, 2015 and except irrigation all the cultural practices were followed uniformly. The observations on growth, physiological parameters, yield parameters and agronomic parameters were observed and data analysis subjected to LSD at $5 \%$ probability level by Panse and Shukatme (1985).

Factor 1: Depth of soil $D_{1} 15 \mathrm{~cm} \mathrm{D}_{2} 30 \mathrm{~cm}$

Factor 2: Moisture depletion percent $\mathrm{M}_{1} 40$ $\% \mathrm{M}_{2} 60 \%$

Factor 3: Water level $\mathrm{W}_{1} 151 \mathrm{~W}_{2} 201$ per plant

\section{Results and Discussion}

With availability of irrigation water dwindling day-by-day, it has become necessary to resort to alternate water saving methods like drip irrigation which provides continuous supply of water in drops right at the root zone of the plant. The scarcity of water is becoming more acute due to erratic and improper distribution of rainfall and faulty water management practices (Bafna et al., 1993). 


\section{Growth parameters}

The data on the growth parameters showing that the different irrigation regimes were significantly influenced the growth and development of jasmine. Growth in terms of plant height and leaf area at 30,60, 90, 120, 150 and 180 days after pruning showed significant difference among different irrigation regimes.

\section{Plant height (cm)}

Among the different irrigation regimes, the plants receiving 20 litre of irrigation water per plant when soil moisture depletion reaches 60 $\%$ at the depth of $30 \mathrm{~cm}$ recorded maximum plant height 81.9, 105.8, 120.0, 131.7, 138.4 and $141.6 \mathrm{~cm}$ at $30,60,90,120,150$ and 180 days after pruning respectively. This was closely followed by the plants receiving 20 litre of water per plant when soil moisture depletion reaches $60 \%$ at $15 \mathrm{~cm}$ depth of soil (78.7, 99.1, 111.0, 121.0, 126.4 and $128.7 \mathrm{~cm}$ at 30,60, 90, 120, 150 and 180 days after pruning respectively). Simultaneously, the flood irrigated plants recorded least values for plant height among the different irrigation regimes (72.0, 79.7, 85.2, 88.4, 89.6 and 90.1 at $30,60,90,120,150$ and 180 days after pruning respectively (Table 1 ).

The linear trend in plant growth might be due to the fact that optimum irrigations maintained most of the root zone at well aerated condition and at adequate soil moisture content there were no fluctuations between wet and dry extremes (Patil and Janawade, 1999). This findings was corresponding with previous results revealed by Taweesaak et al., 2014. Availability of moisture under drip irrigation might have contributed to effective absorption and utilization of nutrients and better proliferation of roots resulting in better growth. Better soil moisture condition may positively contribute for higher solubility and mobility of nutrients which ultimately results into increased mass flow transport of nutrients (Tisdale et al., 1997).

\section{Leaf area}

Irrigation regime of 20 litre of irrigation water per plant when soil moisture depletion reaches $60 \%$ at the soil depth of $30 \mathrm{~cm}$ were recorded highest leaf area of 7.07, 8.14, 9.29, $10.50,11.82$ and $12.84 \mathrm{~cm}^{2}$ at $30,60,90,120$, 150 and 180 days after pruning respectively. This was closely followed by the plants receiving 20 litre of water per plant when soil moisture depletion reaches $60 \%$ at $15 \mathrm{~cm}$ depth of soil $(6.87,7.90,8.99,10.16,10.60$ and $12.32 \mathrm{~cm}^{2}$ at $30,60,90,120,150$ and 180 days after pruning respectively). The flood irrigated plants recorded least values for leaf area among the different irrigation regimes $\left(6.06,6.81,7.53,7.91,8.43\right.$ and $8.62 \mathrm{~cm}^{2}$ at $30,60,90,120,150$ and 180 days after pruning respectively (Table 2). Growth and development in plants are a consequence of excellent coordination of several processes operating at different stages of plant. Reduced irrigation level resulting in water deficit might manifest many changes in plant anatomy such as decrease in size of cells and inter cellular spaces limiting cell division and elongation resulting in overall decrease in plant growth (May and Milthrope, 1962).In the present study the growth of jasmine, as influenced by the various treatments, has been elucidated through plant height and leaf area.

\section{Yield parameters}

Earlier flower bud emergence (24.4 days) and earlier flower bud picking (34.2 days)were observed in the treatment receiving optimum irrigation frequency and control (Flood irrigation) taken maximum days for flower bud emergence and flower bud picking (Table $3)$. 
Fig.1 Effects of different irrigation regimes on yield of flower buds per plant of Jasminum sambac cv. Ramanathapuram Gundumalli

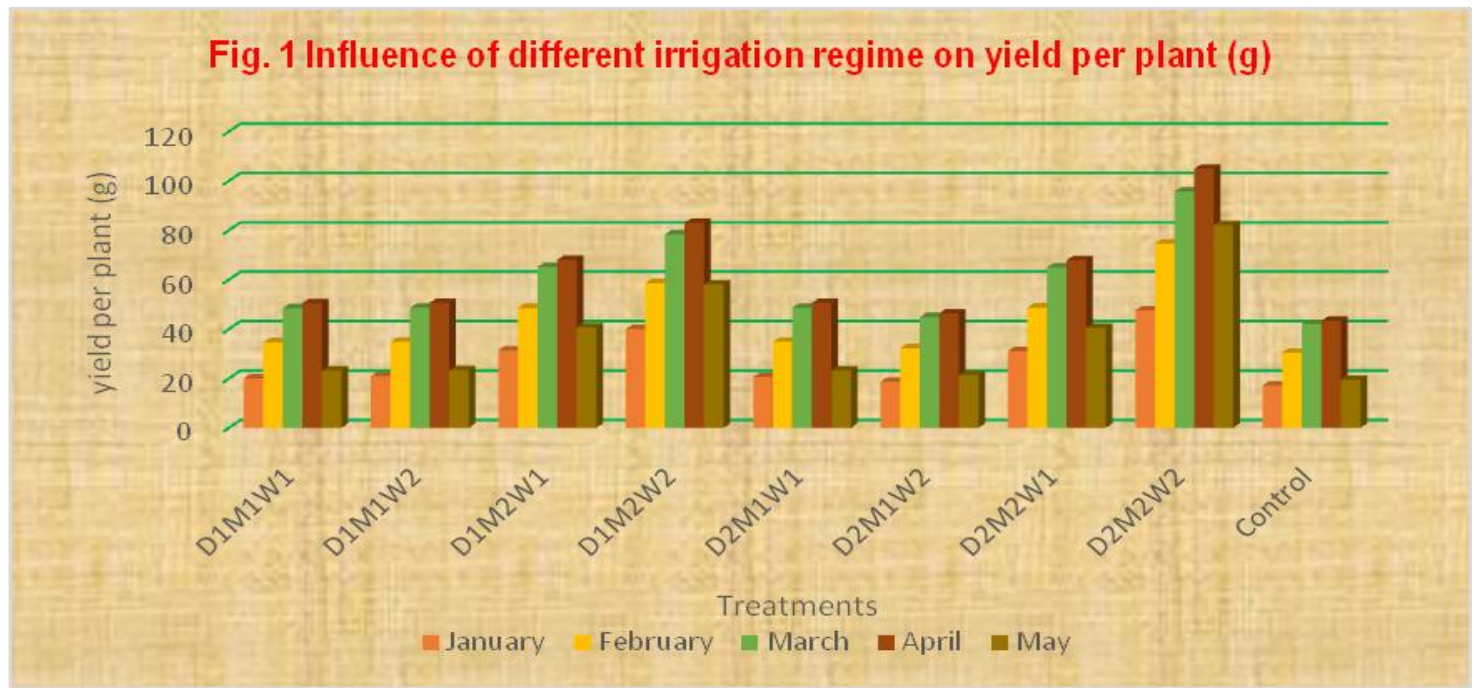

Fig.2 Effects of different irrigation regimes on irrigation frequency and water use efficiency of Jasminum sambac cv. Ramanathapuram Gundumalli

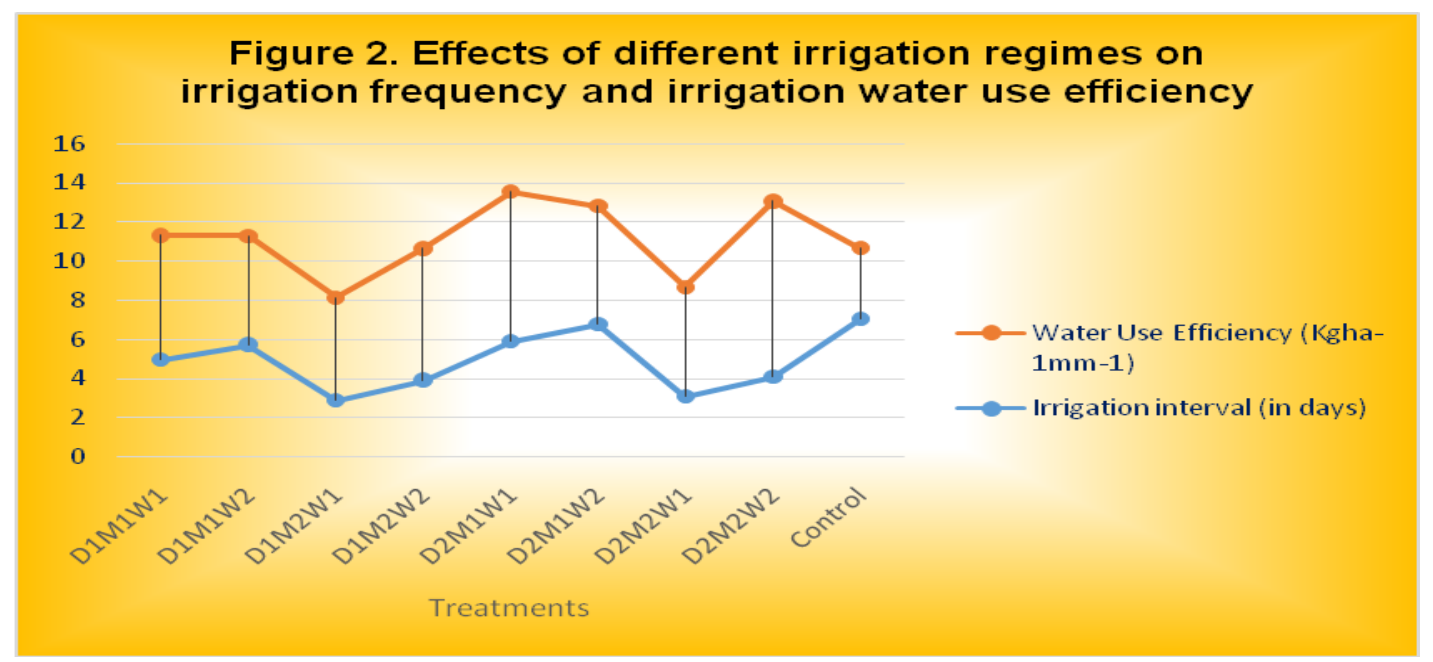

\section{Treatment details}

$\mathrm{D}_{1} \mathrm{M}_{1} \mathrm{~W}_{1}$ - Soil depth $15 \mathrm{~cm}+$ Moisture depletion $40 \%+$ Water level 151 per plant

$\mathrm{D}_{1} \mathrm{M}_{1} \mathrm{~W}_{2}$ - Soil depth $15 \mathrm{~cm}+$ Moisture depletion $40 \%+$ Water level 201 per plant

$\mathrm{D}_{1} \mathrm{M}_{2} \mathrm{~W}_{1}$ - Soil depth $15 \mathrm{~cm}+$ Moisture depletion $60 \%+$ Water level 151 per plant

$\mathrm{D}_{1} \mathrm{M}_{2} \mathrm{~W}_{2}$ - Soil depth $15 \mathrm{~cm}+$ Moisture depletion $60 \%+$ Water level 201 per plant

$\mathrm{D}_{2} \mathrm{M}_{1} \mathrm{~W}_{1}$ - Soil depth $30 \mathrm{~cm}+$ Moisture depletion $40 \%+$ Water level 151 per plant

$\mathrm{D}_{2} \mathrm{M}_{1} \mathrm{~W}_{2}$ - Soil depth $30 \mathrm{~cm}+$ Moisture depletion $40 \%+$ Water level 201 per plant

$\mathrm{D}_{2} \mathrm{M}_{2} \mathrm{~W}_{1}$ - Soil depth $30 \mathrm{~cm}+$ Moisture depletion $60 \%+$ Water level 151 per plant

$\mathrm{D}_{2} \mathrm{M}_{2} \mathrm{~W}_{2}$ - Soil depth $30 \mathrm{~cm}+$ Moisture depletion $60 \%+$ Water level 201 per plant

Control - Flood irrigation once in a week 
Table.1 Effect of different irrigation regime on plant height (cm) of Jasminum sambac cv. Ramanathapuram Gundumalli at 30, 60, 90, 120,150 and 180 days after pruning

\begin{tabular}{|c|c|c|c|c|c|c|c|c|c|c|c|}
\hline \multirow{2}{*}{$\begin{array}{c}\text { Days after } \\
\text { pruning }\end{array}$} & \multirow{2}{*}{ Treatments } & \multicolumn{2}{|c|}{$\mathbf{D}_{1}$} & \multirow{2}{*}{ Mean } & \multicolumn{2}{|c|}{$\mathbf{D}_{2}$} & \multirow{2}{*}{ Mean } & \multicolumn{2}{|c|}{$\mathbf{W} \times \mathbf{M}$} & \multirow{2}{*}{ Mean } & \multirow[t]{2}{*}{ Control } \\
\hline & & $\mathbf{W}_{1}$ & $\mathbf{W}_{2}$ & & $W_{1}$ & $\mathbf{W}_{2}$ & & $\mathbf{W}_{1}$ & $\mathbf{W}_{2}$ & & \\
\hline \multirow{3}{*}{30 DAP } & $\mathbf{M}_{1}$ & 74.6 & 74.7 & 74.7 & 74.8 & 73.6 & 74.2 & 74.7 & 74.2 & 74.4 & \multirow{3}{*}{72.0} \\
\hline & $\mathbf{M}_{2}$ & 76.8 & 78.7 & 77.7 & 76.9 & 81.9 & 79.4 & 76.9 & 80.3 & 78.6 & \\
\hline & Mean & 75.7 & 76.7 & 76.2 & 75.9 & 77.7 & 76.8 & 75.8 & 77.2 & 76.5 & \\
\hline \multirow{3}{*}{60 DAP } & $\mathbf{M}_{1}$ & 89.6 & 89.5 & 89.6 & 89.4 & 84.6 & 87.0 & 89.5 & 87.1 & 88.3 & \multirow{3}{*}{79.7} \\
\hline & $\mathbf{M}_{2}$ & 94.6 & 99.1 & 96.8 & 94.9 & 105.8 & 100.3 & 94.7 & 102.5 & 98.6 & \\
\hline & Mean & 92.1 & 94.3 & 93.2 & 92.1 & 95.2 & 93.7 & 92.1 & 94.8 & 93.4 & \\
\hline \multirow{3}{*}{90 DAP } & $\mathbf{M}_{1}$ & 98.1 & 97.9 & 98.0 & 97.6 & 91.7 & 94.7 & 97.8 & 94.8 & 96.3 & \multirow{3}{*}{85.2} \\
\hline & $\mathbf{M}_{2}$ & 104.7 & 111.0 & 107.8 & 104.6 & 120.0 & 112.3 & 104.7 & 115.5 & 110.1 & \\
\hline & Mean & 101.4 & 104.4 & 102.9 & 101.1 & 105.9 & 103.5 & 101.2 & 105.1 & 103.2 & \\
\hline \multirow{3}{*}{120 DAP } & $\mathbf{M}_{1}$ & 104.0 & 103.8 & 103.9 & 103.7 & 96.6 & 100.2 & 103.9 & 100.2 & 102.1 & \multirow[t]{3}{*}{88.4} \\
\hline & $\mathbf{M}_{2}$ & 111.9 & 121.0 & 116.5 & 111.8 & 131.7 & 121.8 & 111.9 & 126.4 & 119.1 & \\
\hline & Mean & 108.0 & 112.4 & 110.2 & 107.8 & 114.2 & 111.0 & 107.9 & 113.3 & 110.6 & \\
\hline \multirow{3}{*}{150 DAP } & $\mathbf{M}_{1}$ & 107.0 & 106.7 & 106.9 & 106.7 & 98.3 & 102.5 & 106.9 & 102.5 & 104.7 & \multirow[t]{3}{*}{89.6} \\
\hline & $\mathbf{M}_{2}$ & 116.2 & 126.4 & 121.3 & 116.0 & 138.4 & 127.2 & 116.1 & 132.4 & 124.3 & \\
\hline & Mean & 111.6 & 116.6 & 114.1 & 111.4 & 118.3 & 114.9 & 111.5 & 117.5 & 114.5 & \\
\hline \multirow{3}{*}{180 DAP } & $\mathbf{M}_{1}$ & 107.8 & 107.4 & 107.6 & 107.3 & 99.1 & 103.2 & 107.5 & 103.2 & 105.4 & \multirow[t]{3}{*}{90.1} \\
\hline & $\mathbf{M}_{2}$ & 117.3 & 128.7 & 123.0 & 117.2 & 141.6 & 129.4 & 117.2 & 135.2 & 126.2 & \\
\hline & Mean & 112.5 & 118.1 & 115.3 & 112.2 & 120.3 & 116.3 & 112.4 & 119.2 & 115.8 & \\
\hline
\end{tabular}

\begin{tabular}{|c|c|c|c|c|c|c|c|c|}
\hline & & D & $\mathbf{M}$ & $\mathbf{W}$ & DM & MW & DW & DMW \\
\hline \multirow{2}{*}{30 DAP } & SEd & 0.1005 & 0.1005 & 0.1005 & 0.1422 & 0.1422 & 0.1422 & 0.2011 \\
\hline & CD @ 0.05 & 0.2157 & 0.2157 & 0.2157 & 0.3051 & 0.3051 & 0.3051 & 0.4315 \\
\hline \multirow{2}{*}{60 DAP } & SEd & NS & 0.2463 & 0.2463 & 0.3484 & 0.3484 & \multirow{2}{*}{ NS } & 0.4927 \\
\hline & CD@0.05 & NS & 0.5285 & 0.5285 & 0.7474 & 0.7474 & & 1.0569 \\
\hline \multirow{2}{*}{90 DAP } & SEd & 0.1488 & 0.1488 & 0.1488 & 0.2104 & 0.2104 & 0.2104 & 0.2976 \\
\hline & CD@0.05 & 0.3192 & 0.3192 & 0.3192 & 0.4515 & 0.4515 & 0.4515 & 0.6385 \\
\hline \multirow{2}{*}{120 DAP } & SEd & NS & 0.4351 & 0.4351 & 0.6154 & 0.6154 & 0.6154 & 0.8703 \\
\hline & CD@0.05 & INS & 0.9334 & 0.9334 & 1.3201 & 1.3201 & 1.3201 & 1.8669 \\
\hline \multirow{2}{*}{150 DAP } & SEd & 0.2844 & 0.2844 & 0.2844 & 0.4022 & 0.4022 & 0.4022 & 0.5688 \\
\hline & CD@0.05 & 0.6101 & 0.6101 & 0.6101 & 0.8628 & 0.8628 & 0.8628 & 1.2202 \\
\hline \multirow{2}{*}{180 DAP } & SEd & 0.4362 & 0.4362 & 0.4362 & 0.6169 & 0.6169 & 0.6169 & 0.8724 \\
\hline & CD@0.05 & 0.9357 & 0.9357 & 0.9357 & 1.3232 & 1.3232 & 1.3232 & 1.8714 \\
\hline
\end{tabular}


Table.2 Effect of different irrigation regime on leaf area $\left(\mathrm{cm}^{2}\right)$ of Jasminum sambac cv. Ramanathapuram Gundumalli at 30, 60, 90, 120,150 and 180 days after pruning

\begin{tabular}{|c|c|c|c|c|c|c|c|c|c|c|c|}
\hline \multirow{2}{*}{$\begin{array}{c}\text { Days after } \\
\text { pruning }\end{array}$} & \multirow{2}{*}{ Treatments } & \multicolumn{2}{|c|}{$\mathbf{D}_{1}$} & \multirow{2}{*}{ Mean } & \multicolumn{2}{|c|}{$\mathbf{D}_{2}$} & \multirow{2}{*}{ Mean } & \multicolumn{2}{|c|}{$\mathbf{W} \times \mathbf{M}$} & \multirow{2}{*}{ Mean } & \multirow[t]{2}{*}{ Control } \\
\hline & & $\mathbf{W}_{1}$ & $\mathbf{W}_{2}$ & & $W_{1}$ & $\mathbf{W}_{2}$ & & $\mathbf{W}_{1}$ & $\mathbf{W}_{2}$ & & \\
\hline \multirow{3}{*}{30 DAP } & $\mathbf{M}_{1}$ & 6.41 & 6.42 & 6.41 & 6.42 & 6.21 & 6.31 & 6.41 & 6.31 & 6.36 & \multirow{3}{*}{6.06} \\
\hline & $\mathbf{M}_{2}$ & 6.71 & 6.87 & 6.79 & 6.69 & 7.07 & 6.88 & 6.70 & 6.97 & 6.84 & \\
\hline & Mean & 6.56 & 6.65 & 6.60 & 6.56 & 6.64 & 6.60 & 6.56 & 6.64 & 6.60 & \\
\hline \multirow{3}{*}{60 DAP } & $\mathbf{M}_{1}$ & 7.19 & 7.18 & 7.19 & 7.18 & 6.97 & 7.08 & 7.19 & 7.08 & 7.13 & \multirow{3}{*}{6.81} \\
\hline & $\mathbf{M}_{2}$ & 7.63 & 7.90 & 7.76 & 7.62 & 8.14 & 7.88 & 7.62 & 8.02 & 7.82 & \\
\hline & Mean & 7.41 & 7.54 & 7.48 & 7.40 & 7.56 & 7.48 & 7.41 & 7.55 & 7.48 & \\
\hline \multirow{3}{*}{90 DAP } & $\mathbf{M}_{1}$ & 8.05 & 8.04 & 8.05 & 8.04 & 7.74 & 7.89 & 8.05 & 7.89 & 7.97 & \multirow{3}{*}{7.53} \\
\hline & $\mathbf{M}_{2}$ & 8.61 & 8.99 & 8.80 & 8.61 & 9.29 & 8.95 & 8.61 & 9.14 & 8.88 & \\
\hline & Mean & 8.33 & 8.52 & 8.42 & 8.33 & 8.51 & 8.42 & 8.33 & 8.52 & 8.42 & \\
\hline \multirow{3}{*}{120 DAP } & $\mathbf{M}_{1}$ & 8.94 & 8.95 & 8.94 & 8.94 & 8.15 & 8.54 & 8.94 & 8.55 & 8.74 & \multirow{3}{*}{7.91} \\
\hline & $\mathbf{M}_{2}$ & 9.65 & 10.16 & 9.91 & 9.63 & 10.50 & 10.06 & 9.64 & 10.33 & 9.98 & \\
\hline & Mean & 9.29 & 9.56 & 9.42 & 9.28 & 9.32 & 9.30 & 9.29 & 9.44 & 9.36 & \\
\hline \multirow{3}{*}{150 DAP } & $\mathbf{M}_{1}$ & 9.85 & 9.82 & 9.83 & 9.83 & 8.63 & 9.23 & 9.84 & 9.22 & 9.53 & \multirow{3}{*}{8.43} \\
\hline & $\mathbf{M}_{2}$ & 10.73 & 11.38 & 11.06 & 10.72 & 11.82 & 11.27 & 10.72 & 11.60 & 11.16 & \\
\hline & Mean & 10.29 & 10.60 & 10.44 & 10.27 & 10.23 & 10.25 & 10.28 & 10.41 & 10.35 & \\
\hline \multirow{3}{*}{180 DAP } & $M_{1}$ & 10.32 & 10.32 & 10.32 & 10.32 & 8.98 & 9.65 & 10.32 & 9.65 & 9.99 & \multirow{3}{*}{8.62} \\
\hline & $\mathbf{M}_{2}$ & 11.35 & 12.32 & 11.84 & 11.33 & 12.84 & 12.09 & 11.34 & 12.58 & 11.96 & \\
\hline & Mean & 10.84 & 11.32 & 11.08 & 10.83 & 10.91 & 10.87 & 10.83 & 11.12 & 10.97 & \\
\hline
\end{tabular}

\begin{tabular}{|c|c|c|c|c|c|c|c|c|}
\hline & & D & M & $\mathbf{W}$ & DM & MW & DW & DMW \\
\hline \multirow{2}{*}{30 DAP } & SEd & 0.0121 & 0.0121 & 0.0121 & 0.0171 & 0.0171 & 0.0171 & 0.0242 \\
\hline & CD@0.05 & 0.0260 & 0.0260 & 0.0260 & 0.0368 & 0.0368 & 0.0368 & 0.0368 \\
\hline \multirow{2}{*}{60 DAP } & SEd & 0.0200 & 0.0200 & 0.0200 & 0.0283 & 0.0283 & 0.0283 & 0.0400 \\
\hline & CD@0.05 & 0.0429 & 0.0429 & 0.0429 & 0.0607 & 0.0607 & 0.0607 & 0.0859 \\
\hline \multirow{2}{*}{90 DAP } & SEd & 0.0145 & 0.0145 & 0.0145 & 0.0205 & 0.0205 & 0.0205 & 0.0290 \\
\hline & CD@0.05 & 0.0311 & 0.0311 & 0.0311 & 0.0441 & 0.0441 & 0.0441 & 0.0623 \\
\hline \multirow{2}{*}{120 DAP } & SEd & 0.0319 & 0.0319 & 0.0319 & 0.0451 & 0.0451 & 0.0451 & 0.0638 \\
\hline & CD@0.05 & 0.0684 & 0.0684 & 0.0684 & 0.0967 & 0.0967 & 0.0967 & 0.1368 \\
\hline \multirow{2}{*}{150 DAP } & SEd & 0.0271 & 0.0271 & 0.0271 & 0.0384 & 0.0384 & 0.0384 & 0.0543 \\
\hline & CD@0.05 & 0.0582 & 0.0582 & 0.0582 & 0.0824 & 0.0824 & 0.0824 & 0.1165 \\
\hline \multirow{2}{*}{180 DAP } & SEd & 0.0302 & 0.0302 & 0.0302 & 0.0428 & 0.0428 & 0.0428 & 0.0605 \\
\hline & CD@0.05 & 0.0649 & 0.0649 & 0.0649 & 0.0918 & 0.0918 & 0.0918 & 0.1298 \\
\hline
\end{tabular}


Table.3 Effect of different irrigation regime on number days taken for flower bud emergence and first harvesting of Jasminum sambac cv. Ramanathapuram Gundumalli

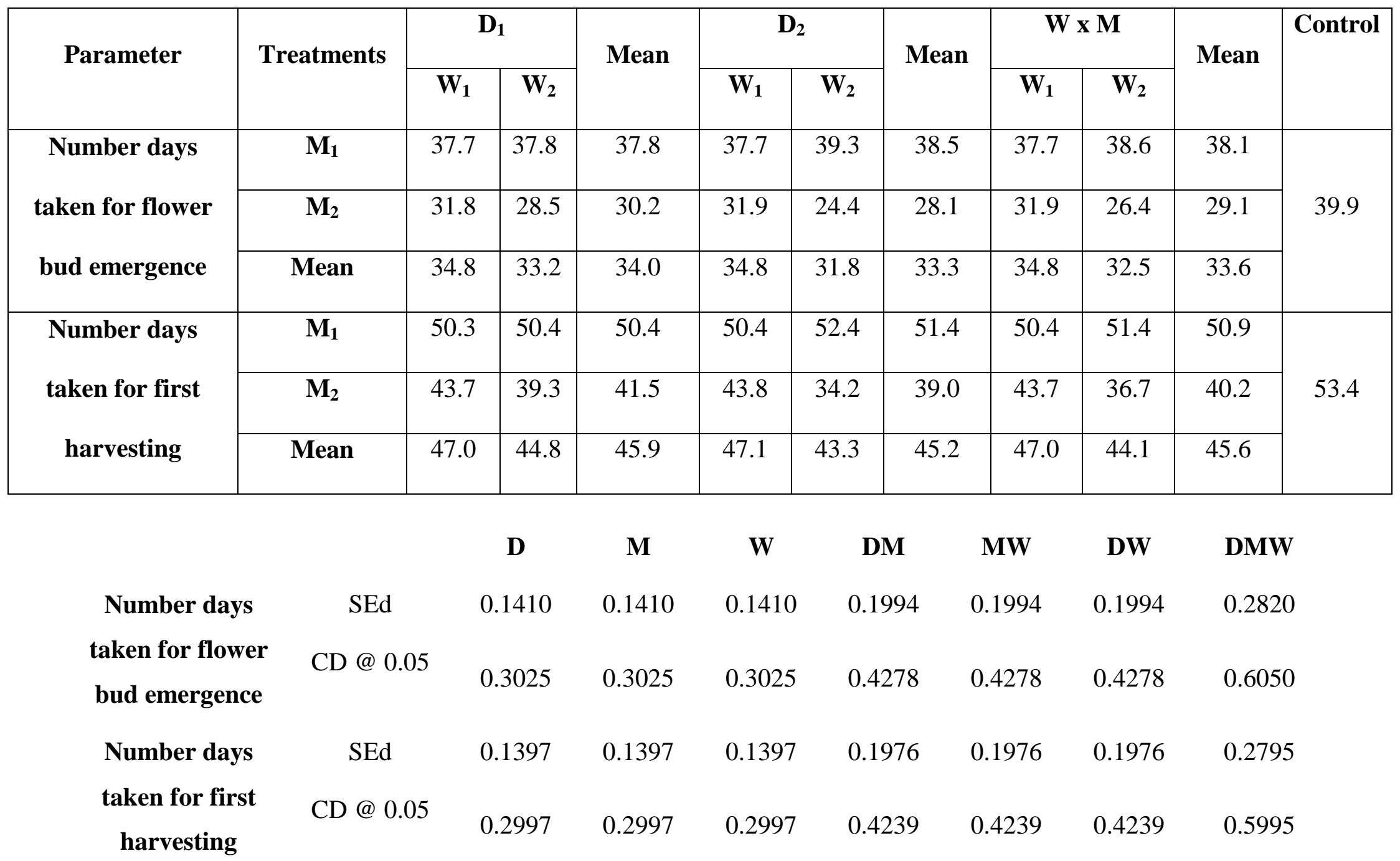


Table.4 Effect of different irrigation regime on yield per plant ofJasminum sambac cv. Ramanathapuram Gundumalli during January, February and March

\begin{tabular}{|c|c|c|c|c|c|c|c|c|c|c|c|}
\hline \multirow{2}{*}{ Period } & \multirow{2}{*}{ Treatments } & \multicolumn{2}{|c|}{$\mathbf{D}_{1}$} & \multirow{2}{*}{ Mean } & \multicolumn{2}{|c|}{$\mathbf{D}_{2}$} & \multirow{2}{*}{ Mean } & \multicolumn{2}{|c|}{$\mathbf{W} \times \mathbf{M}$} & \multirow{2}{*}{ Mean } & \multirow[t]{2}{*}{ Control } \\
\hline & & $\mathbf{W}_{1}$ & $\mathbf{W}_{2}$ & & $\mathbf{W}_{1}$ & $\mathbf{W}_{2}$ & & $\mathbf{W}_{1}$ & $\mathbf{W}_{2}$ & & \\
\hline \multirow{3}{*}{$\begin{array}{c}31 \text { - } 60 \text { DAP } \\
\text { (January) }\end{array}$} & $\mathbf{M}_{1}$ & 20.06 & 20.95 & 20.51 & 20.62 & 18.72 & 19.67 & 20.34 & 19.84 & 20.09 & \multirow{3}{*}{17.15} \\
\hline & $\mathbf{M}_{2}$ & 31.40 & 39.98 & 35.69 & 31.05 & 49.70 & 40.38 & 31.23 & 44.84 & 38.03 & \\
\hline & Mean & 25.73 & 30.47 & 28.10 & 25.84 & 34.21 & 30.02 & 25.78 & 32.34 & 29.06 & \\
\hline \multirow{3}{*}{$\begin{array}{c}61 \text { - } 90 \text { DAP } \\
\text { (February) }\end{array}$} & $\mathbf{M}_{1}$ & 34.63 & 34.88 & 34.76 & 34.81 & 32.26 & 33.54 & 34.72 & 33.57 & 34.15 & \multirow{3}{*}{30.49} \\
\hline & $\mathbf{M}_{2}$ & 48.53 & 58.61 & 53.57 & 48.68 & 74.54 & 61.61 & 48.61 & 66.58 & 57.59 & \\
\hline & Mean & 41.58 & 46.75 & 44.16 & 41.75 & 53.40 & 47.57 & 41.66 & 50.07 & 45.87 & \\
\hline \multirow{3}{*}{$\begin{array}{c}90-120 \text { DAP } \\
(\text { March })\end{array}$} & $\mathbf{M}_{1}$ & 48.45 & 48.60 & 48.53 & 48.61 & 44.86 & 46.74 & 48.53 & 46.73 & 47.63 & \multirow[t]{3}{*}{42.01} \\
\hline & $\mathbf{M}_{2}$ & 65.18 & 78.37 & 71.78 & 64.97 & 95.66 & 80.32 & 65.08 & 87.02 & 76.05 & \\
\hline & Mean & 56.82 & 63.49 & 60.15 & 56.79 & 70.26 & 63.53 & 56.80 & 66.87 & 61.84 & \\
\hline \multirow{3}{*}{$\begin{array}{l}121 \text { - } 150 \text { DAP } \\
\text { (April) }\end{array}$} & $\mathbf{M}_{1}$ & 50.46 & 50.65 & 50.56 & 50.60 & 46.36 & 48.48 & 50.53 & 48.51 & 49.52 & \multirow{3}{*}{43.34} \\
\hline & $\mathbf{M}_{2}$ & 68.03 & 82.99 & 75.51 & 67.84 & 104.84 & 86.34 & 67.94 & 93.92 & 80.93 & \\
\hline & Mean & 59.25 & 66.82 & 63.03 & 59.22 & 75.60 & 67.41 & 59.23 & 71.21 & 65.22 & \\
\hline \multirow{3}{*}{$\begin{array}{c}151 \text { - } 180 \text { DAP } \\
\text { (May) }\end{array}$} & $\mathbf{M}_{1}$ & 23.25 & 23.45 & 23.35 & 23.29 & 21.35 & 22.32 & 23.27 & 22.40 & 22.84 & \multirow{3}{*}{19.46} \\
\hline & $\mathbf{M}_{2}$ & 40.68 & 58.03 & 49.36 & 40.56 & 82.08 & 61.32 & 40.62 & 70.06 & 55.34 & \\
\hline & Mean & 31.97 & 40.74 & 36.35 & 31.93 & 51.72 & 41.82 & 31.95 & 46.23 & 39.09 & \\
\hline
\end{tabular}

$\begin{array}{ccccccccc} & & \text { D } & \text { M } & \text { W } & \text { DM } & \text { MW } & \text { DW } & \text { DMW } \\ \text { 31- 60 DAP } & \text { SEd } & 0.2802 & 0.2802 & 0.2802 & 0.3963 & 0.3963 & 0.3963 & 0.5605 \\ \text { (January) } & \text { CD @ 0.05 } & 0.6011 & 0.6011 & 0.6011 & 0.8502 & 0.8502 & 0.8502 & 1.2023 \\ \text { 61-90 DAP } & \text { SEd } & 0.5761 & 0.5761 & 0.5761 & 0.8148 & 0.8148 & 0.8148 & 1.1523 \\ \text { (February) } & \text { CD @ 0.05 } & 1.2358 & 1.2358 & 1.2358 & 1.7477 & 1.7477 & 1.7477 & 2.4717 \\ \text { 90- 120 DAP } & \text { SEd } & 0.6902 & 0.6902 & 0.6902 & 0.9761 & 0.9761 & 0.9761 & 1.3804 \\ \text { (March) } & \text { CD @ 0.05 } & 1.4805 & 1.4805 & 1.4805 & 2.0937 & 2.0937 & 2.0937 & 2.9610 \\ \text { 121-150 DAP } & \text { SEd } & 0.3976 & 0.3976 & 0.3976 & 0.5623 & 0.5623 & 0.5623 & 0.7952 \\ \text { (April) } & \text { CD @ 0.05 } & 0.8529 & 0.8529 & 0.8529 & 1.2062 & 1.2062 & 1.2062 & 1.7058 \\ \text { 151- 180 DAP } & \text { SEd } & 0.4568 & 0.4568 & 0.4568 & 0.6461 & 0.6461 & 0.6461 & 0.9137 \\ \text { (May) } & \text { CD @ 0.05 } & 0.9800 & 0.9800 & 0.9800 & 1.3859 & 1.3859 & 1.3859 & 1.9600\end{array}$


It could be due to the fact that, optimum irrigation and pruning helps to broaden the $\mathrm{C} / \mathrm{N}$ ratio, thus stimulating flowering and increasing vigour of plant. These results are in line with the findings of Jadhav et al., (2003) and Ghulam et al., (2004) in rose.

Data on flower yield per plant are presented in Table 4 and Figure 1. The maximum yield (49.70, 74.54, 95.66, 104.84 and $82.08 \mathrm{~g}$ ) per plant was recorded by the treatment receiving 201 of irrigation water when moisture depletion reaches $60 \%$ at $30 \mathrm{~cm}$ depth. The lowest yield of 17.15, 30.49, 42.01, 43.34 and $19.46 \mathrm{~g}$ per plant was recorded by flood irrigation (Control) during the month of January 2016, February 2016, March 2016, April 2016 and May 2016 respectively.

This might be attributed to effective utilization of root zone irrigation and fertilizer. Irrigation scheduling based on developmental stage or deficit irrigation is the technique of applying water on a timely and accurate basis to the crop, and is the key to conserving water and improving irrigation performance and sustainability of irrigated agriculture (Mpelasoka et al., 2001).

Behboudian and Mills (1997) defined deficit irrigation as applying less water to the plant at selected stages during the growing season. This approach may save water with little or no negative impact on the final crop yield.

\section{Irrigation frequency and Irrigation Water use efficiency}

From the statistical analysis, the optimum irrigation frequency was 4 days interval and maximum water use efficiency $\left(8.99 \mathrm{kgha}^{-}\right.$ ${ }^{1} \mathrm{~mm}^{-1}$ ) recorded by the plants receiving 20 litre of irrigation water per plant when moisture depletion percentage reaches $60 \%$ at the soil depth of $30 \mathrm{~cm}$ with maximum value for all the yield contributing attributes. The least water use efficiency was recorded by control treatment $\left(3.63 \mathrm{kgha}^{-1} \mathrm{~mm}^{-1}\right)$. Saving of irrigation water was found in all drip irrigation regimes than plants receiving flood irrigation (Figure 2).

Similar findings on water saving by drip irrigation were reported by Ahluwaalia et al., (1993); Bafna et al., (1993b); Pawar et al., (1993) and Ramesh et al., (1994).

During the period of experiment, there was a temperature range between 29.2 to $41.3^{\circ} \mathrm{C}$ and evaporation between the ranges of 1.5 to 4.1 $\mathrm{cm}$. The irrigation water efficiently utilized by the plants which receiving optimum irrigation frequency.

The results obtain from this experiment were showed that optimum irrigation frequency resulted in highest plant growth and flower yield as well as water use efficiency.

The very low and higher irrigation frequency resulted lower yield. This was accorded with study one in gerbera, in which flowering was not influenced by the very low irrigation frequency (Tsirogiannis, 2010).

In the present work different irrigation regimes were tried to standardize the drip irrigation scheduling. Results from this experiment demonstrated that the plants receiving 201 of irrigation when soil moisture depletion reaches $60 \%$ at $30 \mathrm{~cm}$ soil depth can improve the vegetative and flowering characteristics. This treatment were recorded optimum irrigation frequency (4 days interval). This may be attributable to availability of sufficient moisture for better growth and yield of Jasminum sambac.

\section{References}

Ahluwaalia, M.S., Singh,B. and Gill, B.S. 1993. Drip irrigation system - it's hydraulic performance and influence on tomato and 
cauliflower crops. Journal of Water Management 1(1): 6-9.

Bafna, A.M., Daftardar,S.Y. Khade, K.K. Patel, P.V. and Dhotre,R.S. 1993ab. Utilization of nitrogen and water by drip irrigation system. J. Water Manage., 1: 1-5.

Behboudian, M. H. and Mills,T. M. 1997. Deficit irrigation in deciduous orchards. Hortic. Rev. 21: 105-131.

Bhattacharjee, S. K., 1980. Native jasmine of India. Indian Perfumes, 24(3): 126-133.

Ghulam, Z., Muhammad, M., Qasim, M., Jamil, M. and Jalalud-Din, B. 2004. Effect of extent of puring on growth and flowering characteristics of six exotic rose cultivars. Sarhad J. Agric., 20(4): 501-506.

Jacobs, J.M., Mohanty, M.P., Hsu, E-C.and Miller, D. 2004. SMEX02: Field scale variability, time stability and similarity of soil moisture. Remote Sens. Environ. 92(4): 436-446.

Jadhav, V.B., Patil, M. T., Gaikwad, A. M. andPatil,J. 2003. Influence of different pruning levels on growth and flowering of roses under green houses. J. Maharashtra Agric. Univ., 28(3): 313-314.

Jayamma, N., Nagaraj M. Naik and Jagadeesh, K.S. 2014. Influence of Biofertilizer application on growth, yield and quality parameters of Jasmine (Jasminum auriculatum). International Conference on Food, Biological and Medical Sciences (FBMS-2014) Jan. 28-29, 2014 Bangkok (Thailand).

May, L.H. and Milthrope, F.Z. 1962. Drought resistance of crop plants. Fld. Crop Abstracts 23: 171.

Mpelasoka, B. S., Behboudian, M. H. and Mills,T. M. 2001. Effect of deficit irrigation on fruit maturity and quality of 'Braeburn' apple.
Sci. Hortic. 90(3/4): 279-290.

Panse, V.G. and Sukhatme,P.V. 1978. Statistical method for agricultural workers, II Edn. ICAR, New Delhi, India.

Patil, V. S. and Janawade,A.D. 1999. Soil water plant atmosphere relationships. In: Proc. Advances in microirrigation and fertigation. June 21-30. Dharwad. Karnataka :19-32.

Pawar, V.S., Maher, D.P.,Dahiwalker, S.D. and Bhoite,S.D. 1993. Liquid fertilizer effect through drip irrigation on yield and water use efficiency of garlic. J. Water Management 1(1): 10-12.

Ramesh, P., Kailasam, C. and Srinivasan, T.R. 1994. Performance of sugarcane (SccharumofficinarumL.) under surface, drip, subsurface drip (Biwall) and furrow methods of irrigation. J. Agronomy and Crops Sci., 172: 327-341.

SushmaLokhande, NehaChopde, ParinitaWasnik and NehaNehare, 2015. Response of Jasminum sambac (L.) to time and severity of pruning. Plant Archives, 15(2):759-762.

Taweesaak, V., Abdullah,T.L.,Hassan, S.A., Kamarulzaman, N.H. and Yusaoff, W.A.W. 2014. Growth and flowering responses of cut chrysanthemum grown under restricted root volume to irrigation frequency. Hindawi Publishing Corporation Scientific World Journal, ID 254867, 6 pp. http://dx. Doi.org/10.115/2014/254867.

Tisdale, S. L., Nelson, W. L., Beaton, J. D. and Harin, J. L. 1997. Soil fertility and fertilizers. Prentice Hall of India Pvt. Ltd., New Delhi. Pp. 109- 346.

Tsirogiannis, I., Katsoulas, N. and Kittas, C. 2010. Effect of irrigation scheduling on gerbera flower yield and quality. Hort Science, 45(2):265-270.

\section{How to cite this article:}

Kumaresan S. and Jawaharlal M. 2017. Standardization of Drip Irrigation Scheduling for Jasmine (Jasminum sambac Ait. cv. Ramanathapuram Gundumalli) using Theta Probe moisture meter. Int.J.Curr.Microbiol.App.Sci. 6(12): 5385-5394.

doi: https://doi.org/10.20546/ijcmas.2017.612.503 